\title{
Development of SYBR Green I-based polymerase chain reaction for feline bocavirus 1 detection
}

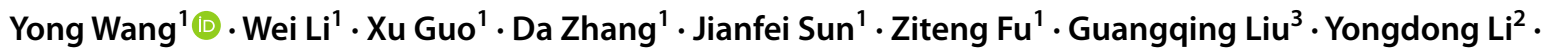 \\ Shudong Jiang ${ }^{1}$
}

Received: 8 September 2020 / Accepted: 1 December 2020 / Published online: 11 January 2021

(c) King Abdulaziz City for Science and Technology 2021

\begin{abstract}
Feline bocavirus 1 (FBoV-1) may be associated with diarrhea in cats. In this study, a SYBR Green I-based quantitative polymerase chain reaction (qPCR) assay was established to detect FBoV-1. The melting curve showed a single melting peak at $83.0^{\circ} \mathrm{C}$. The results of sensitivity showed that the detection limit of the qPCR was $3.87 \times 10^{1}$ copies $/ \mu \mathrm{L}$. Of note, the detection limit of the conventional polymerase chain reaction (cPCR) was $3.87 \times 10^{3}$ copies $/ \mu \mathrm{L}$. The highest intra-assay and inter-assay coefficients of variation (CV\%) were $0.98 \%$ and $1.42 \%$, respectively. The positive detection rate of 128 clinical samples using the qPCR and the cPCR was 7.0\% (9/128) and 4.7\% (6/128), respectively. Taken together, these results indicated that the established qPCR assay has good sensitivity, high specificity, and good reproducibility. Therefore, it could provide support for the rapid and efficient clinical detection of FBoV-1.
\end{abstract}

Keywords Detection $\cdot$ Feline bocavirus $1 \cdot$ SYBR green I qPCR

\section{Introduction}

Bocavirus is a member of the genus Bocaparvovirus, which belongs to the subfamily Parvovirinae, in the family Parvoviridae (Manteufel and Truyen 2008). The virus is a nonenveloped, 5.4-kb, single-stranded DNA virus with three open reading frames (ORFs): ORF1, ORF2, and ORF3, which encode non-structural protein (NS1), viral capsid

Wei Li and Yong Wang contributed equally to this work and considered to be the co-first authors.

Yongdong Li

liyd0551@126.com

Shudong Jiang

jshudong@163.com

1 Anhui Province Key Laboratory of Veterinary Pathobiology and Disease Control, College of Animal Science and Technology, Anhui Agricultural University, Hefei 230036, People's Republic of China

2 Municipal Key Laboratory of Virology, Ningbo Municipal Center for Disease Control and Prevention, Ningbo 315010, People's Republic of China

3 Shanghai Veterinary Research Institute, Chinese Academy of Agricultural Sciences, Shanghai 200241, People's Republic of China proteins (VP1 and VP2), and nuclear phosphoprotein (NP1), respectively (Allander et al. 2005; Lau et al. 2012; Takano et al. 2016; Amimo et al. 2017). Compared with other parvoviruses, the bocavirus ORF3 is an extra ORF between the non-structural and structural-coding regions (Allander et al. 2005; Lau et al. 2012; Takano et al. 2016). So far, the genus Bocaparvovirus includes 25 species: Carnivore bocaparvovirus 1-6, Chiropteran bocaparvovirus 1-4, Lagomorph bocaparvovirus 1, Pinniped bocaparvovirus 1-2, Primate bocaparvovirus 1-2, Rodent bocaparvovirus 1-2, and Ungulate bocaparvovirus 1-8. Bocavirus can infect a wide range of animals, including human (Verbeke et al. 2019), pigs (Mohan Jacob et al. 2018; Shi et al. 2019), canines (Guo et al. 2016), California sea lion (Li et al. 2011), felines (Takano et al. 2016), and gorilla (Kapoor et al. 2010). The virus usually causes severe gastrointestinal and respiratory diseases in young hosts; whereas, subclinical infections are more common in adults (Lefkowitz et al. 2018; Liu et al. 2018).

Feline bocavirus (FBoV) was first detected in Hong Kong in 2012 (Lau et al. 2012). Three types of FBoV have been detected (FBoV-1, FBoV-2, and FBoV-3), corresponding to Carnivore bocaparvovirus 3-5, respectively (Lefkowitz et al. 2018; Liu et al. 2018; Lau et al. 2012). Compared with healthy cats, FBoV-1 is more likely to be detected in cats 
with diarrhea (Yi et al. 2018). In fact, studies have shown that FBoV-1 is closely associated with diarrhea. Co-infections with FBoV-1 and Feline panleukopenia virus (FPLV) are also common, leading to more serious clinical symptoms, such as hemorrhagic enteritis (Piewbang et al. 2019; Liu et al. 2018). Therefore, infection with FBoV-1 poses a health risk to cats.

An efficient identification method for $\mathrm{FBoV}$ can provide a powerful tool for the clinical diagnosis of cats with diarrhea. So far, there are conventional polymerase chain reaction (cPCR) methods and Taqman probe-based methods to detect FBoV (Wang et al. 2020); however, they are not without limitations. cPCR methods are associated with low sensitivity and are time-consuming, while the Taqman probe-based methods require the design and synthesis of specific probes, resulting in high detection prices (Wang et al. 2017). Quantitative real-time polymerase chain reaction (qPCR) has been demonstrated to be very useful for quantifying viral pathogens (Cardoso et al. 2013). In this study, we established a quick and specific SYBR Green I -based qPCR method for FBoV-1 detection, with the aim to aid the clinical diagnosis of FBoV-1 infection.

\section{Materials and methods}

\section{Virus samples and DNA and RNA extraction}

The FBoV-1 AAU01 strain (GenBank number: MT577646) was collected from the feces of cats with hemorrhagic enteritis in an animal hospital in Hefei, Anhui Province, China. Feline Parvovirus (FPV) HF1 strain (GenBank number: MT614366), Feline Herpesvirus (FHV, Fel-O-Vax PCT feline vaccine, Boehringer-Ingelheim), Feline Coronavirus (FCoV) HF1902 strain (GenBank number: MT444152), FBoV-2 HFXA-6 strain (GenBank number: MT633126), FBoV-3 HFTH-22 strain (GenBank number: MT633128), and Feline Astrovirus (FeAstV) AH-1-2020 strain (GenBank number: MN977118), which were preserved in our laboratory, were used for the specificity analysis of the qPCR method. For DNA viruses, DNA was extracted from the feces using the TIANamp Virus DNA/RNA Kit (TIANGEN, Beijing, China) according to the manufacturer's instructions. For RNA viruses, reverse transcription of $100 \mathrm{ng}$ viral RNA sample was carried out using the FastQuant cDNA Kit (TIANGEN) according to the manufacturer's instructions. The DNA/cDNA was stored at $-20^{\circ} \mathrm{C}$ until use.

\section{Primer design}

Compared with the VP1 and NP1 genes, the NS1 gene of FBoV-1 is more conserved (Yi et al. 2018). A pair of specific primers (F-s/R-s) was designed based on the NSI gene of
FBoV-1 using http://bioinfo.ut.ee/primer3-0.4.0/ (Table 1). In addition, primers for amplifying $N S 1$ were designed using Primer Premier 5 software.

\section{Preparation of standard plasmid}

The NS1 gene was amplified by cPCR reaction that comprised $12.5 \mu \mathrm{L}$ of $2 \times$ Premix Taq $^{\circledR}$ Version 2.0 (TaKaRa, Dalian, China), $1 \mu \mathrm{L}$ of the DNA template, $0.4 \mu \mathrm{M}$ forward and reverse primers (primer F-z and R-z), and an appropriate volume of $\mathrm{ddH}_{2} \mathrm{O}$ to obtain a total volume of $25 \mu \mathrm{L}$. The PCR products were first purified, then cloned into pMD19-T vector (TaKaRa), and finally transformed into $E$. coli $\mathrm{DH} 5 \alpha$ competent cells. The recombinant plasmid, named pMD19TNS1, was extracted using the EasyPure ${ }^{\circledR}$ plasmid MiniPrep kit (TransGen Biotech, Beijing, China) and sequenced by the General Biological Company (Anhui, China). The concentration of the extracted plasmid was measured with NANODROP 2000 system (Thermo Fisher Scientific, Waltham, Massachusetts, USA).The plasmid copy number (copies $/ \mu \mathrm{L}$ ) was determined according to the formula: [concentration of plasmids $\left.(\mathrm{ng} / \mu \mathrm{L}) \times 6.02 \times 10^{23}\right] /\left[10^{9} \times\right.$ length of genome (bp) $\times 660 \mathrm{Da} / \mathrm{bp}$ )]. Tenfold dilution of pMD19T-NS1 was prepared and stored at $4{ }^{\circ} \mathrm{C}$.

\section{Optimization and establishment of standard curves}

The prepared pMD19T-NS1 plasmid was used as template in SYBR Green I -based qPCR reactions. All reactions were performed in the CFX96TM Real-Time System (Bio-Rad, CA, U.S.A). Each reaction was of $20 \mu \mathrm{L}$, and contained $10 \mu \mathrm{L} 2 \times$ SuperReal PreMix Plus (Tiangen), $0.6 \mu \mathrm{L}$ of the forward primer (F-s), $0.6 \mu \mathrm{L}$ of the reverse primer (R-s), $1.0 \mu \mathrm{L}$ of standard DNA plasmid, and $7.8 \mu \mathrm{L}$ of RNase-free $\mathrm{ddH}_{2} \mathrm{O}$. The reaction consisted of an initial activation step of $15 \mathrm{~min}$ at $95^{\circ} \mathrm{C}, 40$ cycles of denaturation step of $10 \mathrm{~s}$ at $95{ }^{\circ} \mathrm{C}$, and annealing step of $30 \mathrm{~s}$ at $60{ }^{\circ} \mathrm{C}$. Each reaction was repeated three times.

Table 1 Primer sequences designed in the study

\begin{tabular}{lllc}
\hline Primer & Sequence $\left(5^{\prime}-3^{\prime}\right)$ & Genomic position & $\begin{array}{l}\text { Product } \\
\text { length } \\
\text { (bp) }\end{array}$ \\
\hline F-s & CAAGCTCTATGGTTGCGT & $1623-1642$ & 240 \\
& CA & & \\
R-s & TTGCCACCCAGTGTTTGA & $1843-1862$ \\
& TA & & \\
F-z & TCGGACCTGGAGAAG & $194-212$ & 3360 \\
& AACA & & \\
R-z & CATACCAGCACCGTT & $3341-3359$ & \\
& ACCA & & \\
\hline
\end{tabular}




\section{Sensitivity analysis of SYBR Green I qPCR}

To optimize the SYBR Green I qPCR, a tenfold continuous dilution of the plasmid standard was used to determine the detection limit. The plasmid concentration of $3.87 \times 10^{8} \sim 3.87 \times 10^{1}$ copies $/ \mu \mathrm{L}$ was used to determine the sensitivity of the reaction. Meanwhile, plasmids with the same concentration gradient were used as templates for cPCR detection. The PCR products were electrophoresed on agarose gel and observed under ultraviolet light to determine sensitivity. The detection limit of SYBR Green I qPCR was confirmed based on the Cycle Threshold $(C t)$ value at the highest dilution. Finally, the sensitivity of cPCR and SYBR Green I qPCR was compared.

\section{Specificity analysis of SYBR Green I qPCR}

The established SYBR Green I qPCR was used to simultaneously detect FPV, FHV, FCV, FCoV, FAstV, FBOV-2, FBOV-3, and RNase-free $\mathrm{H}_{2} \mathrm{O}$ to confirm the specificity of this technique.

\section{Reproducibility analysis of SYBR Green I qPCR}

The pMD19T-NS1 plasmid at concentrations of $3.87 \times 10^{8}-3.87 \times 10^{1}$ copies $/ \mu \mathrm{L}$ was used as template to detect the reproducibility of the assay according to the optimized system. The reactions were repeated three times a day for three consecutive days to assess the intra-assay and interassay standard deviations and the repeatability and stability of the method.

\section{Detection in clinical samples}

To demonstrate the clinical applicability of SYBR Green I qPCR, we collected 128 fecal samples of cats from animal hospitals in China. DNA of the samples was extracted using the Qiagen DNA tissue kit (Qiagen) according to the manufacturer's instructions, and stored at $-20{ }^{\circ} \mathrm{C}$. The extracted DNA was subjected to both cPCR and SYBR Green I qPCR detection methods.

\section{Results}

\section{Construction of the SYBR Green I qPCR standard curve}

The recombinant plasmid pMD19T-NS1 was diluted serially in concentrations ranging from $3.87 \times 10^{8}$ to $3.87 \times 10^{1}$ copies $/ \mu \mathrm{L}$, and used for SYBR Green I qPCR. The SYBR Green I qPCR amplification curve was constructed under optimal conditions. The $C t$ value linearly correlated with the logarithm of plasmid copy number $\left(R^{2}=0.999\right)($ Fig. 1b); the correlation stand curve equation was: $Y=-3.447 \times+34.123$ (Fig. 1b), the amplification efficiency (E) was $95.0 \%$, and the melt peak temperature was $83.0^{\circ} \mathrm{C}$ (Fig. 1c).

\section{Sensitivity of the SYBR Green I qPCR}

The sensitivity of both PCR methods was determined by measuring the minimum detection limit of standard plasmids with tenfold serial dilutions $\left(3.87 \times 10^{8}-3.87 \times 10^{1}\right.$ copies/ $\mu \mathrm{L})$. The minimum detection limit of the SYBR Green I qPCR was $3.87 \times 10^{1}$ copies $/ \mu \mathrm{L}$, and that for gel electrophoresis using products of SYBR Green I qPCR was $3.87 \times 10^{3}$ copies $/ \mu \mathrm{L}$. Thus, the SYBR Green I qPCR was found to be more sensitive than cPCR (Fig. 2).

\section{Specificity of the SYBR Green I qPCR}

We used six viruses (including FPV, FHV, FCoV, FAstV, FBoV-2, and FBoV-3) to verify the specificity of the SYBR Green I PCR. In addition, RNase-free $\mathrm{H}_{2} \mathrm{O}$ was used as a negative control. Only the pMD19T-NS1 group demonstrated a high-intensity fluorescent signal with a $C t$ value lower than 35 (Fig. 3). FHV and the negative control did not show specific amplification. Additionally, although the curves of FPV, FBoV-2, FAstV, FCoV, and FBoV-3 were higher than the threshold, their $\mathrm{Ct}$ values are all greater than 35 (Fig. 3). Therefore, the SYBR Green I qPCR established in the study is highly specific.

\section{Reproducibility of the SYBR Green I qPCR}

The intra-assay and the inter-assay coefficients of variation (CV\%) ranged from $0.24 \%$ to $0.98 \%$ and $0.54 \%$ to $1.42 \%$, respectively (Table 2). These results indicated that the SYBR Green I qPCR is reproducible.

\section{Screening of clinical specimens by SYBR Green I PCR}

The SYBR Green I qPCR established in this study and cPCR were simultaneously used to test 128 clinical samples. The positive detection rate of the samples by SYBR Green I qPCR was $7.0 \%$ (9/128), whereas that by cPCR was $4.7 \%$ (6/128) (Table 3). These results indicate that the SYBR Green I qPCR was more sensitive than cPCR.

\section{Discussion}

Viral diarrhea is a very common disease in cats and is often considered to be caused by FPV infection (Stuetzer and Hartmann 2014; Zhang et al. 2019). However, with advances in scientific research, many more diarrhea-related viruses

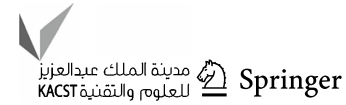



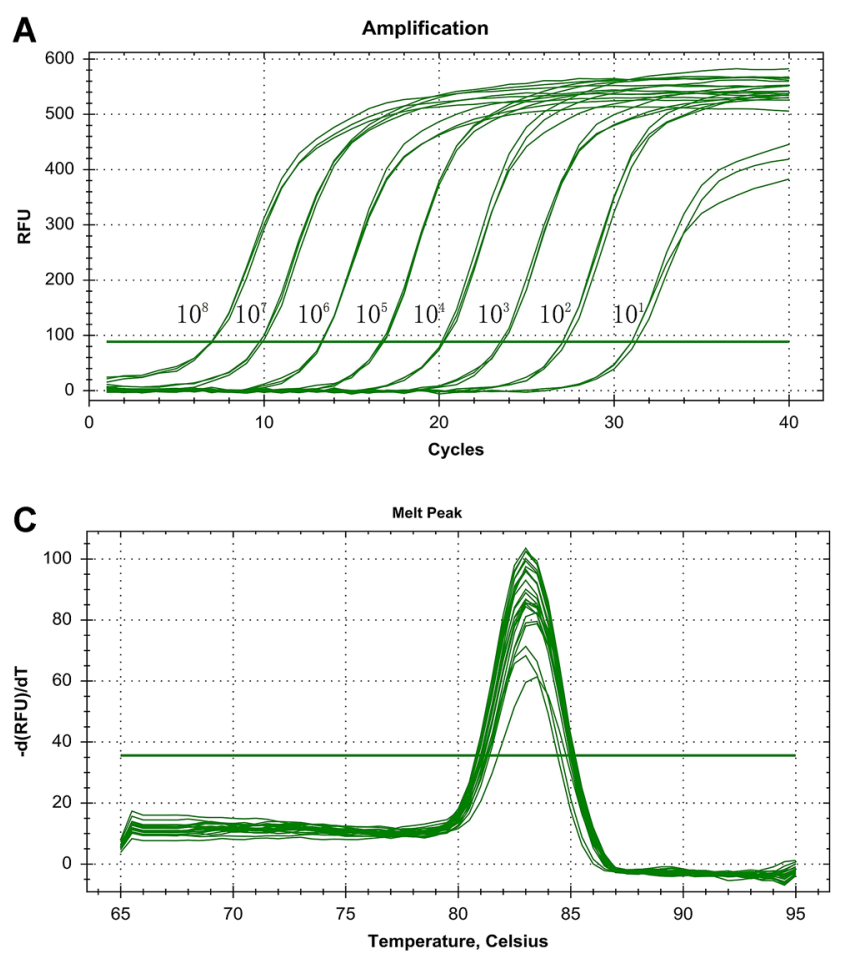

Fig. 1 a Amplification curves of SYBR Green-based qPCR. The concentration of the plasmid pMD19T-NS1 ranged from $3.87 \times 10^{8}$ to $3.87 \times 10^{1}$ copies $/ \mu \mathrm{L}$. b Standard curves of $\mathrm{qPCR}$ based on SYBR Green I for FBoV-1 detection. The plasmids $\left(3.87 \times 10^{8}-3.87 \times 10^{1}\right.$ copies $\left./ \mu \mathrm{L}\right)$ were used to establish the standard curve: Equation: $y=-3.447 \times \lg$ [virus copies] +34.123 ; correlation coefficient: $R^{2}=0.999$; reaction efficiency: $E=95.0 \%$. c
B

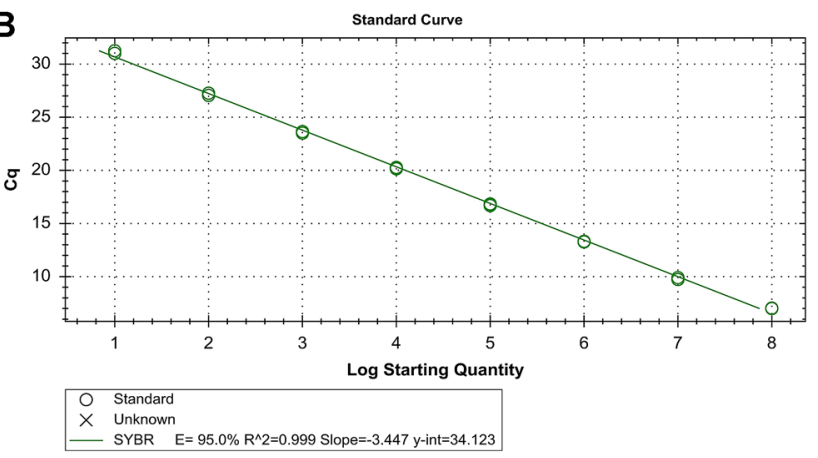

D

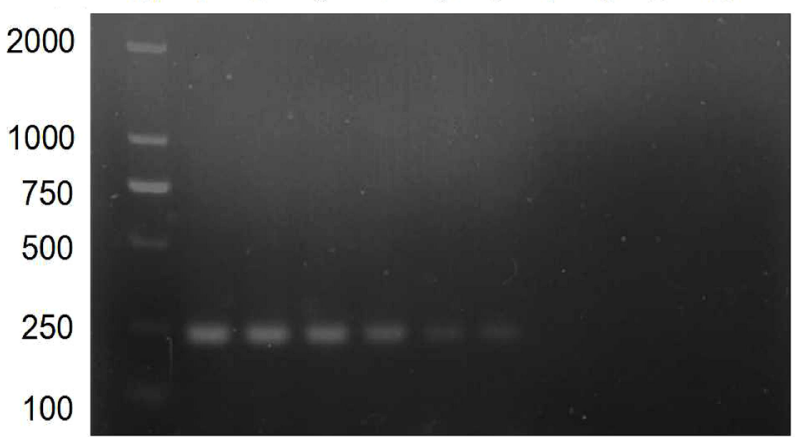

Melting curve analysis of qPCR based on SYBR Green I. The Tm of FBoV-1 qPCR was $83.0^{\circ} \mathrm{C}$. d The gel electropherogram results of conventional PCR. DNA marker of $2000 \mathrm{bp}$ was used. The tenfold serial dilutions of pMD19T-NS1 plasmids ranging from $3.87 \times 10^{8}$ to $3.87 \times 10^{1}$ copies/ $\mu \mathrm{L}$ in lanes $1-8$, respectively; lane 9 contains the negative control
Fig. 2 Amplification curve of qPCR based on SYBR Green I used for sensitivity analysis. Amplification was carried out with tenfold dilutions of standard plasmids ranging from $10^{8}$ to $10^{1}$ copies

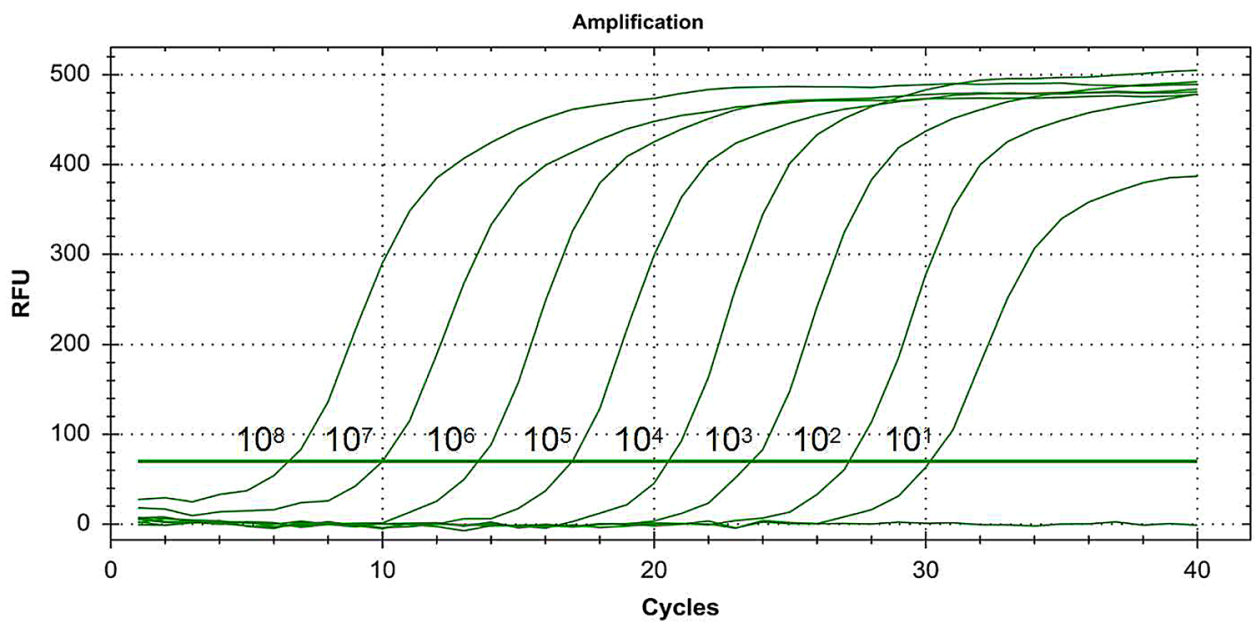

have been reported, such as FeAstV and Feline Kobuvirus (FeKoV) (Cho et al. 2014; Lu et al. 2018). The FBoV, first discovered in Hong Kong in 2012, is also considered to be a diarrhea-related virus (Lau et al. 2012; Piewbang et al. 2019; $\mathrm{Li}$ et al. 2020). The detection rate of $\mathrm{FBoV}$ in cats with diarrhea in northeast China was reported to be as high as $33 \%$, which is the highest detection rate of FBoV-1 so far (Yi et al. 2018). In this study, we aimed to establish a more rapid and sensitive method for detecting FBoV-1 using SYBR Green I-based qPCR technique.

Compared to cPCR methods, real-time PCR assays have several advantages, such as being rapid, allowing 
Fig. 3 Specificity analysis of the SYBR Green I qPCR for FBoV-1. Only the pMD19TNS1 group demonstrated a high-intensity fluorescent signal with a $C t$ value lower than 35 . FHV and the negative control did not show specific amplification. Additionally, although the curves of FPV, FBoV-2, FAstV, $\mathrm{FCoV}$, and FBoV-3 were higher than the threshold, their $C t$ values are all greater than 35

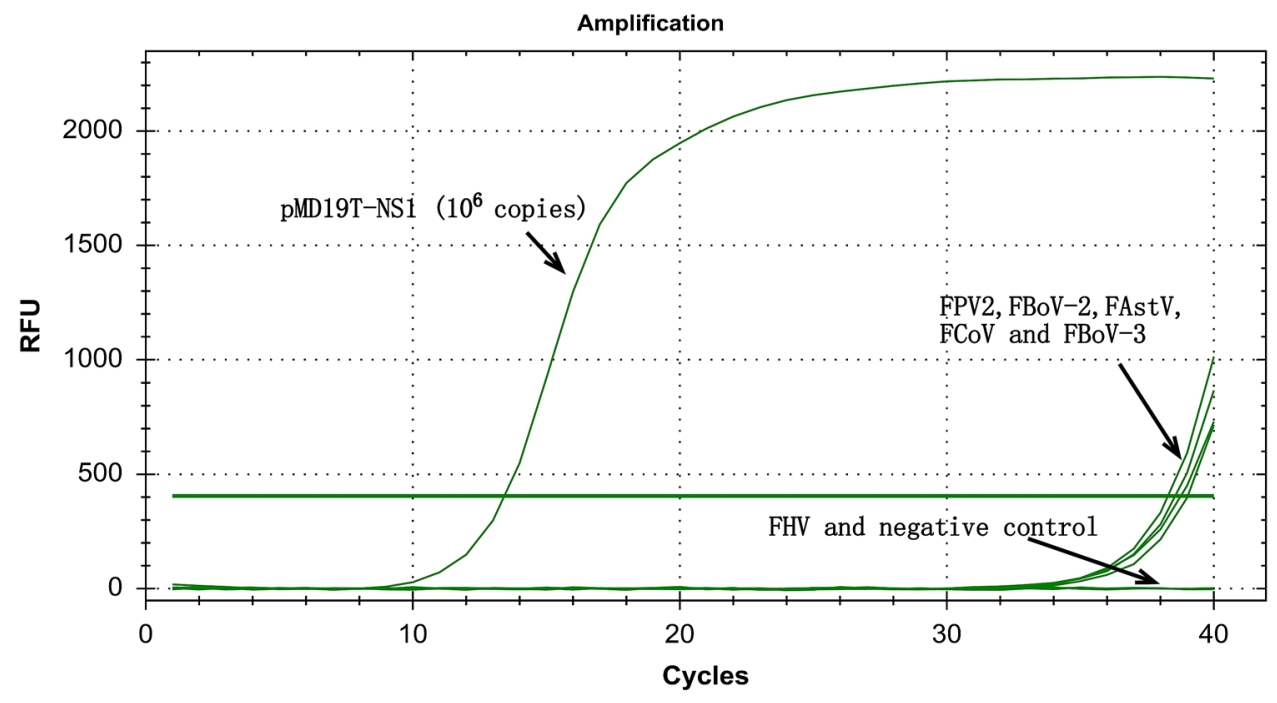

\begin{tabular}{llllllll}
\hline $\begin{array}{l}\text { Copy number of FBoV-1 } \\
(\text { copies/ } \mu \mathrm{L})\end{array}$ & \multicolumn{3}{l}{ Intra-assay variation } & & \multicolumn{3}{l}{ Inter-assay variation } \\
\cline { 2 - 3 } & Mean & SD & CV $(\%)$ & & Mean & SD & CV (\%) \\
\hline $3.87 \times 10^{8}$ & 6.94 & 0.04 & 0.51 & & 6.92 & 0.07 & 0.98 \\
$3.87 \times 10^{7}$ & 9.73 & 0.10 & 0.98 & & 9.85 & 0.14 & 1.42 \\
$3.87 \times 10^{6}$ & 13.24 & 0.03 & 0.24 & & 13.29 & 0.10 & 0.74 \\
$3.87 \times 10^{5}$ & 16.71 & 0.08 & 0.45 & & 16.93 & 0.11 & 0.64 \\
$3.87 \times 10^{4}$ & 20.17 & 0.06 & 0.28 & & 20.29 & 0.12 & 0.57 \\
$3.87 \times 10^{3}$ & 23.54 & 0.08 & 0.32 & & 23.76 & 0.15 & 0.63 \\
$3.87 \times 10^{2}$ & 27.08 & 0.11 & 0.42 & & 27.19 & 0.17 & 0.61 \\
$3.87 \times 10^{1}$ & 31.03 & 0.15 & 0.48 & & 31.34 & 0.17 & 0.54 \\
\hline
\end{tabular}

Table 2 Repeatability analysis of SYBR Green I qPCR
Table 3 Screening of clinical specimens by SYBR Green I qPCR

\begin{tabular}{lclc}
\hline Region & Amount & \multicolumn{2}{l}{ Number of positive samples } \\
\cline { 3 - 4 } & & Conventional PCR & SYBR green I qPCR \\
\hline Hefei & 51 & $3 / 51(5.9 \%)$ & $4 / 51(7.8 \%)$ \\
Nanjing & 20 & $1 / 20(5.0 \%)$ & $2 / 20(10.0 \%)$ \\
Shanghai & 23 & 0 & $1 / 23(4.3 \%)$ \\
Ma' anshan & 19 & $1 / 19(5.3 \%)$ & $1 / 19(5.3 \%)$ \\
Bozhou & 15 & $1 / 15(6.7 \%)$ & $1 / 15(6.7 \%)$ \\
Total & 128 & $6 / 128(4.7 \%)$ & $9 / 128(7.0 \%)$ \\
\hline
\end{tabular}

quantitative measurement, and exhibiting high sensitivity and specificity (Mackay et al. 2002). SYBR Green I qPCR assays are more sensitive and faster than serological diagnosis (Luo et al. 2018). Moreover, compared with the TaqMan probe method, the SYBR Green I qPCR is based on the detection of fluorescent signals emitted by the fluorescent chimeric dye SYBR Green I, which eliminates the need to design and synthesize specific probes; therefore, the experimental steps are much simpler, and the assay is cheaper (Wang et al. 2017). In previous reports, FBoV has often been detected in domestic cats living in constant contact with other cats, e.g., in animal shelters (Li et al. 2020). Using other methods, such as the TaqMan probe method, higher costs would be involved in the testing of $\mathrm{FBoV}$ in numerous domestic cats. Therefore, the SYBR Green I qPCR method established in the study can provide a more suitable approach for the large-scale detection of FBoV based on its low cost and high efficiency.

Previous reports indicate that the $N S 1$ gene of $\mathrm{FBoV}$ is conserved (Yi et al. 2018). In this study, two pairs of primers located in the NSI gene were designed; one was used to amplify the entire NS1 gene for plasmid construction and the other was used for detection of FBoV-1. After optimizing the reaction conditions, a SYBR Green I-based qPCR for FBoV-1 detection method was established. The amplification efficiency of the method was $95.0 \%$ and correlation coefficient was $0.999\left(R^{2}=0.999\right)$. The detection limit of SYBR Green I qPCR was $3.87 \times 10^{1}$ copies $/ \mu \mathrm{L}, 100$ times that of cPCR, and similar to that of the TaqMan method $\left(4.57 \times 10^{1}\right.$ copies $\left./ \mu \mathrm{L}\right)$ established in our laboratory (Wang et al. 2020). In addition, FBoV-2, FBoV-3, and four other common feline viruses were tested and the results showed 
that the established assay had good specificity for FBoV-1. Repeatability experiment results showed that the intra-assay and inter-assay coefficients of variation were lower than $3 \%$, suggesting good reproducibility. The results of clinical sample detection showed that the positive rate of CPCR was $4.7 \%$ $(6 / 128)$, whereas that of SYBR Green I-based qPCR was $7.0 \%$ (9/128), indicating that the established method was more reliable than cPCR in the detection of clinical samples.

In summary, we developed a SYBR Green I-based qPCR assay for FBoV-1 detection and quantification that shows high sensitivity, specificity and repeatability. This method is suitable for diagnosis, epidemiological monitoring, and quantitative study of FBOV-1 infection in clinical samples.

Acknowledgments We would like to thank Editage (www.editage.cn) for English language editing.

Author contributions WL, XG, DZ, JS were involved in performing experiments. WL and GL were involved in analyzing data and designing experiments. WL, YW, SJ and YL wrote the manuscript. All authors have read and approved the final manuscript.

Funding Funding of the study came from Ningbo Health Branding Subject Fund (No. ppxk2018-10), key research project of national science and technology (No. 2016YFD0501003), State Key Laboratory of Genetically Engineered Veterinary Vaccines (No. AGVSKLZD-202010) and the Science and Technology Promotating Agriculture Innovation Project of Shanghai (No. 2019 No.3-3).

\section{Compliance with ethical standards}

Conflict of interest All authors have declared that there was no competing interests existing in the study.

Ethical standards All experiments were conformed to the ethical standards of Anhui Agricultural University.

\section{References}

Allander T, Tammi MT, Eriksson M, Bjerkner A, Tiveljung-Lindell A, Andersson B (2005) Cloning of a human parvovirus by molecular screening of respiratory tract samples. ProcNatlAcadSci USA 102(36):12891-12896

Amimo JO, Njuguna J, Machuka E, Okoth E, Djikeng A (2017) First complete genome sequences of porcine bocavirus strains from East Africa. Genome Announc 5:14

Cardoso TC, Silva-Frade C, Taparo CV, Okamura LH, Flores EF (2013) Validation of a reference control for an SYBR-Green fluorescence assay-based real-time PCR for detection of bovine herpesvirus 5 in experimentally exposed bovine embryos. Mol Cell Probes 27(5-6):237-242

Cho YY, Lim SI, Kim YK, Song JY, Lee JB, An DJ (2014) Molecular characterisation and phylogenetic analysis of feline astrovirus in Korean cats. J Feline Med Surg 16(8):679-683

Guo D, Wang Z, Yao S, Li C, Geng Y, Wang E, Zhao X, Su M, Wei S, Wang X, Feng L, Chang YF, Sun D (2016) Epidemiological investigation reveals genetic diversity and high co-infection rate of canine bocavirus strains circulating in Heilongjiang province, Northeast China. Res Vet Sci 106:7-13
Kapoor A, Mehta N, Esper F, Poljsak-Prijatelj M, Quan PL, Qaisar N, Delwart E, Lipkin WI (2010) Identification and characterization of a new bocavirus species in gorillas. PLoS ONE 5(7):e11948

Lau SKP, Woo PCY, Yeung HC, Teng JLL, Wu Y, Bai R, Fan RYY, Chan KH, Yuen KY (2012) Identification and characterization of bocaviruses in cats and dogs reveals a novel feline bocavirus and a novel genetic group of canine bocavirus. J Gen Virol 93(Pt 7):1573-1582

Lefkowitz EJ, Dempsey DM, Hendrickson RC, Orton RJ, Siddell SG, Smith DB (2018) Virus taxonomy: the database of the international committee on taxonomy of viruses (ICTV). Nucleic Acids Res 46(D1):D708-D717

Li L, Shan T, Wang C, Cote C, Kolman J, Onions D, Gulland FM, Delwart E (2011) The fecal viral flora of California sea lions. J Virol 85(19):9909-9917

Li Y, Gordon E, Idle A, Altan E, Seguin MA, Estrada M, Deng X, Delwart E (2020) Virome of a feline outbreak of diarrhea and vomiting includes bocaviruses and a novel chapparvovirus. Viruses 12:5

Liu C, Liu F, Li Z, Qu L (2015) Liu D (2018) First report of feline bocavirus associated with severe enteritis of cat in Northeast China. J Vet Med Sci 80(4):731-735

Lu G, Zhang X, Luo J, Sun Y, Xu H, Huang J, Ou J, Li S (2018) First report and genetic characterization of feline kobuvirus in diarrhoeic cats in China. TransboundEmerg Dis 65(5):1357-1363

Luo Q, Chen B, Xu J, Ma W, Lao C, Li Y, Tan J, Tang Y, Huang C, Liu W, Chen Z (2018) Development of a SYBR green II real-time polymerase chain reaction for the clinical detection of the duck-origin goose parvovirus in China. Intervirology 61(5):230-236

Mackay IM, Arden KE, Nitsche A (2002) Real-time PCR in virology. Nucleic Acids Res 30(6):1292-1305

Manteufel J, Truyen U (2008) Animal bocaviruses: a brief review. Intervirology 51(5):328-334

Mohan Jacob D, Lee CY, Arshad SS, Selvarajah GT, Bande F, Ong BL, Ooi PT (2018) First molecular detection of porcine bocavirus in Malaysia. Trop Anim Health Prod 50(4):733-739

Piewbang C, Kasantikul T, Pringproa K, Techangamsuwan S (2019) Feline bocavirus-1 associated with outbreaks of hemorrhagic enteritis in household cats: potential first evidence of a pathological role, viral tropism and natural genetic recombination. Sci Rep 9(1):16367

Shi QK, Zhang JL, Gu WY, Hou LS, Yuan GF, Chen SJ, Fan JH, Zuo YZ (2019) Seroprevalence of porcine bocavirus in pigs in north-central China using a recombinant-NP1-protein-based indirect ELISA. Arch Virol 164(9):2351-2354

Stuetzer B, Hartmann K (2014) Feline parvovirus infection and associated diseases. Vet J 201(2):150-155

Takano T, Takadate Y, Doki T, Hohdatsu T (2016) Genetic characterization of feline bocavirus detected in cats in Japan. Arch Virol 161(10):2825-2828

Verbeke V, Reynders M, Flore K, Vandewal W, Debulpaep S, Sauer K, Cardoen F, Padalko E (2019) Human bocavirus infection in Belgian children with respiratory tract disease. Arch Virol 164(12):2919-2930

Wang Y, Yang K, Bai C, Yin D, Li G, Qi K, Wang G, Li Y (2017) Development of a SYBR Green I real-time PCR for the detection of the orf virus. AMB Express 7(1):21

Wang Y, Sun J, Guo X, Zhang D, Cui Y, Li W, Liu G, Li Y, Jiang S (2020) TaqMan-based real-time polymerase chain reaction assay for specific detection of bocavirus- 1 in domestic cats. Mol Cell Probes 10:101647

Yi S, Niu J, Wang H, Dong G, Zhao Y, Dong H, Guo Y, Wang K, Hu G (2018) Detection and genetic characterization of feline bocavirus in Northeast China. Virol J 15(1):125

Zhang Q, Niu J, Yi S, Dong G, Yu D, Guo Y, Huang H, Hu G (2019) Development and application of a multiplex PCR method for the simultaneous detection and differentiation of feline panleukopenia virus, feline bocavirus, and feline astrovirus. Arch Virol 164(11):2761-2768 